Cahiers d'études africaines

198-199-200|2010

50 ans

\title{
La culture visuelle au sud du Sahara
}

Visual culture in the South of Sahara

Jean-Paul Colleyn

\section{OpenEdition}

Journals

Édition électronique

URL : https://journals.openedition.org/etudesafricaines/16474

DOI : 10.4000/etudesafricaines. 16474

ISSN : $1777-5353$

Éditeur

Éditions de l'EHESS

Édition imprimée

Date de publication : 20 novembre 2010

Pagination : 1055-1065

ISBN : 978-2-7132-2252-8

ISSN : 0008-0055

Référence électronique

Jean-Paul Colleyn, "La culture visuelle au sud du Sahara », Cahiers d'études africaines [En ligne],

198-199-200 | 2010, mis en ligne le 02 janvier 2013, consulté le 22 avril 2022. URL : http://

journals.openedition.org/etudesafricaines/16474; DOI : https://doi.org/10.4000/etudesafricaines.

16474

Ce document a été généré automatiquement le 22 avril 2022

(c) Cahiers d'Études africaines 


\title{
La culture visuelle au sud du Sahara
}

\author{
Visual Culture in the South of Sahara
}

Jean-Paul Colleyn

1 Le but de cet article n'est pas de faire l'historique des images en Afrique ni des images de l'Afrique, mais de montrer, à grands traits, comment les images sont en quelque sorte les témoins toujours vivants et toujours actifs d'une histoire. C'est un terrain glissant car on sait combien les images laissent la voie libre à des interprétations divergentes et dans ce domaine, les enquêtes de réception n'en sont qu'à leurs balbutiements. Pourtant, dans l'Afrique d'aujourd'hui, il ne fait guère de doute que toute une culture visuelle compte pour beaucoup dans le façonnage des opinions, des attitudes, des valeurs, du pilotage à vue de la vie quotidienne auquel les citoyens sont assignés. Sont concernés ici tous les médiums et tous les supports : les affiches, les posters, la peinture des rues (y compris sur les conteneurs et les véhicules), les graffitis, les tissus et les pagnes, la bande dessinée, la peinture, la sculpture, la photographie, les films et la vidéo, sans oublier ces " performances » qui sont tout à la fois des pièces de théâtre, des concerts et des expositions de peinture (Barber 1987). Certaines images ne font que passer, d'autres se stabilisent en symboles durables par leur forme, plus variables dans leurs contenus. Les clichés ont la vie dure, mais on sait que la reproduction d'un message visuel va rarement sans transformation, aussi bien au niveau de sa fabrication et de sa transmission qu'au niveau de sa réception. L'objectif n'est pas de faire le point sur un sujet ethnographique bien précis; il est plutôt d'ordre programmatique : il s'agit de poser en sujet d'étude, l'extraordinaire ferment culturel que constituent les images dans une Afrique confrontée à de multiples défis.

2 Clarifions d'abord l'usage du mot image, qui est éminemment polysémique, mais dont plusieurs sens nous intéressent ici. Selon une première acception, les images sont des objets concrets qui servent de support à une représentation (dessin, peinture, sculpture, photographie, images animées [film ou vidéo]). Ces images matérielles renvoient donc à autre chose qu'elles-mêmes, et cette autre chose peut être de deux ordres : il peut s'agir d'un référent dans le monde empirique, ou d'une représentation mentale (une idée, une illusion, un souvenir, un fantasme). Enfin, l'image peut être une figure de rhétorique fondée sur l'analogie (la métaphore, la métonymie). La notion 
d'image renferme presque toujours une tension entre deux pôles : celui de la neutralité, de l'objectivité, de la description et celui de l'imaginaire à laquelle elle invite et dont on la charge. Ces deux pôles ne sont jamais faciles à isoler tant l'image prête à la bévue, à l'illusion d'optique (n'oublions pas qu'imago a la même racine qu'imitare, imiter). Bien des nuances de notre vocabulaire courant révèlent cette tension: un cliché est une reproduction résultant d'un procédé mécanique ou chimique, mais c'est aussi une représentation banale, usée, illusoire, naïve. Les usages lexicaux admettent de nombreuses connotations : l'imagerie médicale a toutes les apparences de la science objective, mais l'imagerie populaire renferme un jugement fondé sur un sentiment de supériorité.

\section{L'exotisme en question}

3 La notion d'exotisme reste extrêmement difficile à théoriser. De nombreux intellectuels africains n'aiment guère les africanistes et les ethnographes, qu'ils soupçonnent de vouloir enfermer l'Afrique dans un éternel passé. La catégorie " film ethnographique " ne peut plus se défendre aujourd'hui qu'à condition de l'entendre comme fondée sur une description minutieuse de la vie quotidienne en situation réelle, et de la débarrasser de ses connotations héritées du racisme scientifique de l'époque coloniale, notamment son approche a-historique. La locution «Afrique traditionnelle » n'a pas grand sens si l'on considère l'intensité des bouleversements historiques que n'a cessé de connaitre le continent aussi loin que l'on peut remonter dans le temps. Si la critique saïdienne vise juste - l'africanisme pouvant faire l'objet des mêmes critiques que l'orientalisme (Mudimbe 1988), il est également arrogant de rejeter dans le passé des pratiques artistiques contemporaines, sous le prétexte qu'elles semblent devoir fort peu aux influences occidentales et aux religions de salut. D'autant que la frontière entre ce qui serait traditionnel (depuis toujours l'objet de révisions n'en déplaisent aux nostalgiques de la pureté perdue) et ce qui serait moderne, est aujourd'hui poreuse (Strother 1998; Vogel 1991). On ne compte plus les foires ou les festivals (de masques, de marionnettes, de danse, d'arts plastiques, de poésie, de musique) qui s'organisent en Afrique et ailleurs sur la scène globalisée des échanges internationaux. Le même sculpteur peut en janvier tailler un masque pour sa société villageoise et en février un autre masque pour le marché international. Il n'en est pas pour autant rétrograde en janvier et aliéné en février. Henry John Drewal (1996) a brillamment montré comment l'image "traditionnelle» de Mami Wata s'inscrit dans un circuit qui passe par Hambourg, l'Asie, le delta du Niger, New York et Cuba.

4 La musique highlife du Ghana, qui remonte aux années 1920, mêle les influences des rythmes osibisaba (introduits par les Fante du sud du pays), du Kaiso caribéen, du foxtrot européen, de la musique dagomba du Liberia, des genres ashiko et goombe de Sierra Leone et (plus récemment) de la guitare congolaise (Malaquais 2000).

Une tension demeure entre la négation universaliste des différences postulées a priori (avec le danger d'assimiler l'Autre d'autorité) et le respect des différences (avec le danger d'adopter une posture altérisante). Ne pas voir le semblable dans l'autre et nier ses appartenances propres sont les deux revers de la médaille postcoloniale. Mais la métaphore de la médaille est imparfaite, car le jeu de regards est plus complexe. Fathima Tobing Rony (1996), par exemple, appelle le troisième ceil l'expérience qui consiste à être l'objet d'un regard ethnographique et de se voir soi-même à travers le 
regard du premier monde. Sans doute tout groupe humain a-t-il besoin d'un certain degré d'exotopie, d'une certaine confrontation transculturelle. Comme on le sait, l'identité culturelle provient d'une relation, d'un échange de regards, non d'une ontologie.

\section{Des images imposées...}

6 On dit souvent que l'armée, l'Église et le capitalisme ont été les trois piliers de la colonisation, mais dans l'appropriation colonialiste du monde, toutes les techniques d'enregistrement et de représentation ont joué leur rôle: l'écriture, le dessin, la photographie et le cinéma. L'Autre délibérément construit comme Autre, a été visité, exploré, mesuré, mis en fiches, cartographié, mais surtout, mis en images. Au moment même où leurs formes spécifiques d'art plastique étaient systématiquement accaparées, les Africains se voyaient autoritairement redéfinis de l'extérieur. Depuis la conquête, la vie culturelle de l'Afrique peut être lue comme une longue lutte de reconquête de ses propres images; une lutte qui s'appuie nécessairement sur l'appropriation des techniques européennes; n'oublions pas que dès 1850, la mode des albums-photos s'était déjà répandue dans les villes côtières d'Afrique. Après le long traumatisme colonial, l'art africain, quels que soient ses moyens d'expression, ne pouvait être qu'un art engagé. Il fit et fait encore partie de ce que Georges Balandier avait appelé les reprises d'initiative. Mais les différentes formes de créativité ne se réduisent pas à l'engagement politique, elles correspondent aussi à des stratégies de survie. Bogumil Jewsiewicki (2003) note qu'au Congo, alors que le Blanc s'appliquait à capter les images $\mathrm{du}$ monde congolais - en dessinant, photographiant, mesurant, classant et collectionnant -, les Congolais captaient les images du sien pour les réinterpréter à leur manière (Fabian 1996). Plusieurs auteurs ont retracé l'histoire de la photographie, du cinéma et de la télévision africains, de l'époque coloniale à l'époque actuelle, tant en ce qui concerne la production et les techniques que l'esthétique (Bakari \& Cham 1996; Diawara 1992; Enwezor \& Zaya 1996; Harding 2003; Harrow 2007; Larouche 1991; Murphy 2000; Pfaff 1988; Ukadike 1994). Le cinéma est né en plein triomphe de l'évolutionnisme, une théorie indéfendable scientifiquement (ce n'est pas faute d'avoir essayé), mais qui a perduré et perdure même encore. Le cinéma d'exploration s'inscrit consciemment ou non dans cette matrice idéologique. À noter que le «film d'exploration » ou le « film de voyage » n'ont pas totalement disparu : ils se manifestent sur le mode néocolonial, dans des émissions de télé-réalité où l'on parachute une star du showbiz au milieu des "sauvages", mais aussi sous forme de réappropriations locales. Organisé par l'association Ibn Batouta, le Festival du film d'exploration et de documentaire, se veut, par exemple, un tremplin pour la promotion de la culture et du septième art au Maroc. Au Kenya, la promotion des safaris s'appuie sur les populations locales: les Maasaï et les Samburu jouent habilement de leurs images de guerriers pacifiés, pour bénéficier de la manne du tourisme. Les Dogon en font de même au Mali. L'adoption du cinéma par les créateurs africains ne s'est pas faite facilement. Pendant l'époque coloniale, tandis que le marché international était dominé par les tarzaneries diverses, les agences gouvernementales et les missionnaires tournaient des films à des fins de propagande et d'éducation, surtout dans les domaines sanitaires et dans l'instruction civique. Les Africains n'avaient pratiquement pas accès à la réalisation cinématographique et n'avaient guère le droit de s'y exprimer librement, tant les autorités se méfiaient d'éventuels messages subversifs. En revanche, la photographie, 
qui n'est pas ipso facto un moyen de communication de masse, s'est diffusée plus rapidement. Les œuvres de Mama Casset, Seydou Keita, Malick Sidibé, Samuel Fosso, Philip Kwame Apagya n'ont rien de frontalement politique, mais elles témoignent des aspirations à la modernité de la jeunesse africaine des années 1950, 1960 et 1970.

\section{...Aux images libérées}

7 Après l'indépendance, petit à petit, les élites africaines ont assimilé les techniques de la cinématographie occidentale pour exprimer leurs propres visions du monde. Les cinéastes prirent conscience de la valeur éducative des films et réagirent contre les modèles dominants du cinéma occidental (Diawara 1992 : 101). L'espoir révolutionnaire des lendemains des indépendances donna naissance à ce que l'on a appelé «the Third Cinema " (par analogie au Tiers-Monde) d'inspiration clairement politique (Solanas \& Getino 1976 ; Gabriel 1982). Les efforts des cinéastes africains aboutirent à la création des festivals de Ouagadougou (Fespaco) en 1969 et de Mogadisho, en 1980. Les thèmes les plus fréquemment abordés étaient la critique du colonialisme et de l'Occident, des traditions rétrogrades (polygamie, mariages forcés, mutilations sexuelles, chefferies abusives, maraboutisme), les tensions entre ville et campagne, tradition et modernité, ainsi que tous les problèmes sociaux de l'heure : l'exode rural, l'émigration, l'exil, la corruption, la répression politique (Boughédir 1996). Les différentes réunions internationales de cinéastes africains et les publications qui s'en sont fait l'écho n'ont cessé, année après année, de souligner le besoin impérieux de sortir le cinéma africain de son ghetto élitiste et de montrer des images positives de l'Afrique. En même temps, pour certains cinéastes, la mère-patrie n'était plus un lieu référentiel idéal, mais une contrée désarticulée par le pouvoir colonial et ravagée par la corruption néocoloniale ou postcoloniale, le terme diaspora servant souvent d'euphémisme à l'exil plus ou moins forcé (Hondo 1987 ; Naficy 1999). D'autres idéalisaient la vie rurale comme lieu source malheureusement perverti par un prétendu progrès, d'autres encore, laissaient libre cours à ce qu'Edgar Morin appelait, dans un autre contexte, une " esthétique du désir ", où l'on retrouvait pêle-mêle la musique, les mobylettes, les fringues (la sape), les coiffures "afro", les night-clubs, etc. Ce n'est donc que progressivement que le cinéma est parvenu à dépasser l'opposition binaire tradition-modernité.

\section{Eillères et critique}

Les œuvres d'art contemporaines sont souvent étudiées d'un point de vue culturel, politique ou idéologique plutôt que sur le plan esthétique. Certains efforts ont été faits pour dépasser cette vision conservatrice. Bien des auteurs avaient déjà fait remarquer que l'art religieux et l'art de cour africains étaient analysés comme représentatifs d'un lieu, d'un pays, d'une tradition et surtout d'une ethnie plutôt que comme le produit d'une recherche de formes. L'exposition The Short Century, d'Okwui Enwezor, au Musée d'art contemporain de Chicago en 2001, relevait le défi audacieux de reconstruire une histoire institutionnelle de l'art moderne africain, tout en contestant les canons de cet art tels qu'ils dominent la scène internationale aujourd'hui. Dans l'étude des productions cinématographiques africaines, de nombreux critiques adoptent un paradigme national ou linguistico-culturel - l'Afrique francophone (souvent réduite à l'AOF), l'Afrique anglophone (l'Afrique du Sud faisant l'objet d'un traitement séparé), l'Afrique lusophone, l'Afrique subsaharienne (souvent réduite à une portion), etc. - 
plutôt qu'une approche par thèmes ou par approches cinématographiques. Le paradigme national ou par aire culturelle néglige la complexité de l'identité des créateurs et le phénomène de diaspora. Qu'en est-il, par exemple, d'un réalisateur sénégalais formé à Moscou ou d'un réalisateur éthiopien formé et résidant à Los Angeles? Dans le même ordre d'idée, le film La Genèse de Cheick Oumar Sissoko (1999) est-il plus malien que judéo-chrétien ou vice-versa? Le film Ran, d'Akira Kurosawa (1985), est-il shakespearien ou japonais ? Dans l'étude des formes cinématographiques, souvent, la notion d'auteur prend le pas sur d'autres considérations également importantes comme les conditions de production des œuvres (sous-entendu aussi les conditions posées par les producteurs). Pour le réalisateur ghanéen John Akomfrah, le cinéma africain est potentiellement un cinéma sans frontières (Givanni 1995). Il faut en effet sortir des interprétations culturalistes et des obsessions biographiques pour étudier les films pour ce qu'ils sont: des œuvres contemporaines où se mêlent comme partout l'argent, la sexualité, le pouvoir et la religion dans une logique de «branchements » entre cultures, pour reprendre une métaphore de Jean-Loup Amselle (2001). À Dakar, le gâteau de mariage d'El Hadji Abdou Kader Byèye, est une énorme pièce montée parisienne (Xala, de Sembène Ousmane 1974); la vieille Ramatou corrompt les habitants de Kolobane avec des instruments électroménagers (Hyènes, de Djibril Diop Mambéty 1992), le roi des esprits porte des lunettes noires et un bonnet de laine (Nelisita, de Rui Duarte 1982).

\section{L'espoir d'une production indépendante}

9 Malgré ses difficultés, le cinéma africain atteint assez rapidement sa pleine maturité esthétique. Comme bien des cinémas dans le monde, il reste tiraillé entre les missions éducatives et politiques qu'il se donne et ses aspirations commerciales, mais il se montre inventif et audacieux dans ses procédés rhétoriques. Par rapport à la fiction, le documentaire reste le parent pauvre: un secteur sans argent, qui ne permet pas de gagner sa vie, à moins de travailler pour le secteur éducatif des services gouvernementaux. Mais si le cinéma africain peut revendiquer fièrement sa filmographie, il faut tout de même reconnaître que celle-ci tient du miracle, qu'elle est relativement peu vue en Afrique (où les salles de cinéma ont fermé les unes après les autres) et qu'elle semble condamnée au succès d'estime. Sur le plan économique, ce cinéma n'a pas réussi à s'enraciner comme secteur commercial et culturel indépendant de financements et de réseaux de distribution extérieurs (Diawara 1992). Les festivals consacrés au cinéma africain se portent infiniment mieux que le cinéma africain luimême! La seule grande exception, mais elle doit être prise au sérieux, c'est le boom de la vidéo domestique nigériane, à partir des années 1990. En l'espace de quelques années, ces vidéos ont détrôné les westerns, les films de Kung Fu et les mélodrames indiens naguère si populaires en Afrique. L'esprit d'autonomie des vidéastes nigérians tranche avec le recours aux aides du ministère français de la Coopération dans les pays francophones, un dispositif qui instaura une politique des auteurs féconde sur le plan artistique, mais coupée du public de masse. Les vidéos de Nollywood ne doivent rien à personne, elles fonctionnent sur de petits budgets, avec du matériel semiprofessionnel, elles recherchent leur scénario dans les faits-divers et les rumeurs populaires, elles mélangent allègrement le local et le global, les vivants et les fantômes, le fantasmatique et le quotidien et recherchent avant tout le succès. Elles nous plongent dans un univers sous la pression constante des pulsions et désirs individuels, 
des contraintes collectives et du jugement par un pouvoir transcendant. Si peu réalistes qu'elles soient, elles rendent bien compte de la crise rampante que vivent les habitants des mégapoles africaines.

10

La culture ne s'élabore pas seulement dans les institutions officielles - la presse (écrite et audiovisuelle), les grandes écoles, les théâtres -, elle n'est pas seulement le fait des personnalités connues dont les frasques, faits et gestes sont largement médiatisés, elle est aussi constituée de petites actions ironiques (blagues, slogans, proverbes inventés, caricatures, graffitis, chansons satiriques, danses) faites par les sans-(porte-)voix dans les quartiers populaires des villes africaines. Les peintres, les caricaturistes, les chansonniers apportent souvent sans imprésarios ni maison de production leurs précieuses alluvions d'images et de sons. L'élucidation de la magie propre à l'image, j'emploie volontairement un signifiant flottant à la mode -, qui repose sur les relations encore insuffisamment explorées entre cognition et émotions, passe par le langage verbal qui de ce fait la trahit. Comment traduire une impression physique autant qu'intellectuelle en mots? C'est une difficulté majeure : le seul métalangage possible évacue irrémédiablement les images et les sons au profit des seuls mots. Le mystère du « pouvoir des images » reste donc presque entier, bien qu'essayer de s'y retrouver soit un chantier d'une importance cruciale.

\section{BIBLIOGRAPHIE}

AMSELLE, J.-L., 2001, Branchements. Anthropologie de l'universalité des cultures, Paris, Flammarion. BAKARI, I. \& CHAM, M., 1996, African Experiences of Cinema, London, British Film Institute.

BARBER, K., 1987, « Popular Arts in Africa », African Studies Review, 30 (3) : 1-78.

BOUGHÉDIR, F., 1996, « A Cinema Fighting for its Liberation », in M. MARTIN (ed.), Cinemas of the Black Diaspora. Diversity, Dependence and Oppositionality, Detroit, Wayne State University Press : 11-117. DIAWARA, M., 1992, Black African Cinema : Politics and Culture, Bloomington, Indiana University Press.

DREWAL, H. J., 1996, « Mami Wata Shrines : Exotica and the Construction of the Self », in M.-J. AMOLDI, C. GEARY \& K. HARDIN (eds.), African Material Culture, Bloomington, Indiana University Press : 308-333.

ENWEZOR, O. \& ZAYA, O., 1996, « Colonial Imagery, Tropes of Disruption : History, Culture and Representation in the Works of African Photographers ", in O. ENWEZOR ET AL. (eds.), In/Sight : African Photographers, 1940 to the Present, Catalogue of the Exhibition, New York, S. R. Guggenheim Museum. 
FABIAN, J., 1996, Remembering the Present : Painting and Popular History in Zaire, Berkeley, University of California Press.

-, 1998, Moments of Freedom : Anthropology and Popular Culture, Charlottesville, University Press of Virginia.

FREEDBERG, D., 1989, The Power of Images : Studies in the History and Theory of Response, Chicago, University of Chicago Press.

GABRIEL, T. H., 1982, Third Cinema in the Third World: The Aesthetic of Liberation, Ann Arbor, Umi Research Press («Studies in cinema »).

GIVANNI, J., 1995, « Dream Aloud : an Interview with John Akomfrah », Sight and Sound, 5 (9), September : 37-39.

HARDING, F., 2003, « Africa and the Moving Image : Television, Film and Video ", Journal of African Cultural Studies, 16 (1), Special Issue, « Focusing on the Media in and about Africa » : 69-84.

HARRow, K. W., 2007, Postcolonial African Cinema : From Political Engagement to Postmodernism, Bloomington, Indiana University Press.

HASSAN, S. M., 1995, « The Modernist Experience in African Art : Visual Expressions of the Self and Cross-Cultural Aesthetics », Nka: Journal of Contemporary African Art, $2: 30-33,72$.

HONDO, A. M., 1987, « The Cinema of Exile », in J. D. H. DownING (ed.), Film and Politics in the Third World, New York, Autonomedia : 69-76.

JEWSIEWICKI, B., 1995, Cheri Samba: The Hybridity of Art, Westmount, Quebec, Galerie Amrad African Art Publications.

-, 1999, A Congo Chronicle : Patrice Lumumba in Urban Art, New York, Museum for African Art.

-, 2003, Mami Wata. La peinture urbaine au Congo, Paris, Gallimard (« Le temps des images »).

LAROUCHE, M. (dir.), 1991, Films d'Afrique, Montréal, Guernica.

MALAQUAIS, D., 2000, Review of the Exhibition Hollywood Icons, Local Demons : Ghanaian Popular Paintings by Mark Anthony, by M. Gilbert, American Anthropologist, New Series, 102 (4) : 870-877.

MUdimbe, V. Y., 1988, The Invention of Africa : Gnosis, Philosophy and the Order of Knowledge, Bloomington, Indiana University Press.

MURPHY, D., 2000, « Africans Filming Africa : Questioning Theories of an Authentic African Cinema ", Journal of African Cultural Studies, Taylor \& Francis, Ltd : 239-249.

NAFICY, H., 1999, « Between Rocks and Hard Places : The Interstitial Mode of Production in Exilic Cinema ", in H. NAFICY (ed.), Home, Exile, Homeland : Film, Media, and the Politics of Place, London, Routledge : 125-147.

NUNLEY, J. W., 1987, Moving with the Face of the Devil : Art and Politics in Urban West Africa, Urbana, University of Illinois Press.

PFAFF, F., 1988, Twenty-five Black African Filmmakers : A Critical Study, with Filmography and Biobibliography, Westport, CN, Greenwood Press.

PHILLIPS, R. \& STEINER, C., 1999, Unpacking Culture : Art and Commodity in Colonial and Post-Colonial Worlds, Berkeley, University of California Press.

RONY, F. T., 1996, The Third Eye : Race, Cinema, and Ethnographic Spectacle, Durham, Duke University Press. 
SCHMIDT, N., 1994, Sub-Saharan African Films and Filmmakers 1987-1992 : An Annotated Bibliography, London, Hans Zell Publishers.

SOlAnAS, F. \& GETINO, O., 1976, « Towards a Third Cinema », in B. NICHols (ed.), Movies and Methods. An Anthology, Berkeley, University of California Press : 44-64.

STEINER, C., 1994, African Art in Transit, Cambridge, Cambridge University Press.

STROTHER, Z., 1998, Inventing Masks : Agency and History in the Art of the Central Pende, Chicago,

University of Chicago Press.

UKADIKE, N. F., 1994, Black African Cinema, Berkeley, University of California Press.

VOGEL, S. M. (ed.), 1991, Africa Explores : $20^{\text {th }}$ Century African Art, New York, Center for African Art.

\section{FILMOGRAPHIE}

DUARTE, R., 1982 Nelisita, Angola, 65 minutes, $35 \mathrm{~mm}$ noir et blanc. Production : Laboratorio National de Cinema.

KURoSAWA, A., 1985 Ran, Japon, 2 h 42, 35 mm couleurs. Scénario : Akira Kurosawa, Hideo Oguni, Masato Ide, d'après William Shakespeare (Le roi Lear).

MAMBÉTY, D. D., 1992 Hyènes, Sénégal, 110 minutes, 35 mm couleurs. Production : ADR

Productions, Maag Daan Film \& TV, Thelma Film AG.

SEMBÈNE, O., 1974 Xala, Sénégal, 128 minutes, 35 mm couleurs. Production : Filmi Doomireew, SNC. SIssoко, C. O., 1999 La Genèse, Mali, 102 minutes, 35 mm couleurs. Production : Centre national de production cinématographique (CNPC), Kora Films. Distribution : Cinémathèque Afrique (Cultures France).

\section{RÉSUMÉS}

Résumé

L'article s'attache à montrer comment les images "sur » ou « de » l'Afrique au sud du Sahara quel que soit leur support - sont les témoins toujours vivants et toujours actifs d'une histoire en train de se faire. Des notions comme «image", "film ethnographique» et "Afrique traditionnelle » sont passées au crible de la critique. L'analyse de l'image est toujours sujette à tension entre universalisme et relativisme, mais depuis la conquête coloniale, la vie culturelle de l'Afrique peut être vue comme une longue lutte de reconquête de ses propres images. Les créateurs africains soulignent le besoin impérieux de sortir le cinéma africain de son ghetto élitiste, mais en même temps, pour certains cinéastes, la mère-patrie n'apparaît plus comme un lieu référentiel idéal. De son côté, la critique s'efforce de sortir des interprétations culturalistes et des obsessions biographiques pour étudier les films pour ce qu'ils sont: des œuvres contemporaines où se mêlent comme partout l'argent, la sexualité, le pouvoir et la religion dans une logique transculturelle.

Abstract

The paper shows how the images of Africa, produced by outsiders as well as produced by insiders, are living traces of history. Complex notions such as "image", "ethnographic film", "traditions" have to be deconstructed. Image analysis is always subjected to a tension between universalism and relativism, but since the colonial conquest, the cultural life in Africa can be seen as a struggle 
for its own representations. African artists keep insisting on the need for breaking the elitist ghetto in which African cinema is trapped, but in the same time the motherland has ceased to appear as an ideal. The cultural critique tries to overcome the culturalist bias and the biographic illusions, to interpret films as cultural products where money, sexuality, power, and religion interact in a transcultural context.

INDEX

Keywords : Nollywood, Art, Cinema, Exoticism, Image

Mots-clés : Nollywood, art, cinéma, exotisme, image

\section{AUTEUR}

JEAN-PAUL COLLEYN

Centre d'études africaines, École des hautes études en sciences sociales, Paris. 